\title{
Role of Physiotherapy in Burn and Plastic Surgery
}

\author{
Md Monoarul Haque* \\ Bangladesh Physiotherapy Association, Bangladesh
}

Submission: October 26, 2016; Published: October 31, 2016

*Corresponding author: Md Monoarul Haque, Publication Secretary, Bangladesh Physiotherapy Association, Bangladesh, Tel: +8801628402482

; Email: monoarmunna@yahoo.com

\section{Editorial}

Physiotherapy is a branch of medical science which has widespread applicability, affectivity and impact. Physiotherapist can practice independently or act as a member of multidisciplinary team. In emergency situation as like sports physiotherapy is the first contact treatment which has immediate effect and recovery. Not only that it has long term effect also i.e. rehabilitation. After orthopedic surgery and stroke patients must go rehabilitative protocol. As like other branch of medical sciences physiotherapy has immense role in burn and plastic surgery. Burn affected patients are increasing due to various reason day by day. Besides cleft palate, cleft lip is also seen frequently. In the past there was no individual burn hospital in our country but now there is a Government burn and plastic surgery hospital in Dhaka. High dependency unit, indoor and outdoor facility-all are there but there is no post for physiotherapist. After burn tendency to develop contracture is common. Physiotherapy should be start as early as possible after tissue repair by surgical management to prevent contracture development and make patient functional as much as possible.

Oedema development is another phenomenon of burn injured patients. Here physiotherapist can play role. Physiotherapist should aware about respiratory complications of patients. Chest physiotherapy is an effective way to minimize chest complications. Assessment of individual patient is necessary. Knowledge regarding accident to rehabilitation should be sound. Physiotherapist should be skillful regarding burn patient management. He should keep in mind about type of burn, surgical procedure, pain status and psychological state of patients. Instead of general approach case specific approach is desirable. Research on post-operative physiotherapy can be done. To minimize post-operative complications and to reduce distressful life of patients-physiotherapy has a key role both in short and long term outcome of burn patients and to ensure this appointment of physiotherapist in Government hospital is a demand of time. Policy maker and concern authority should think about role of physiotherapy in case of burn injured patents.

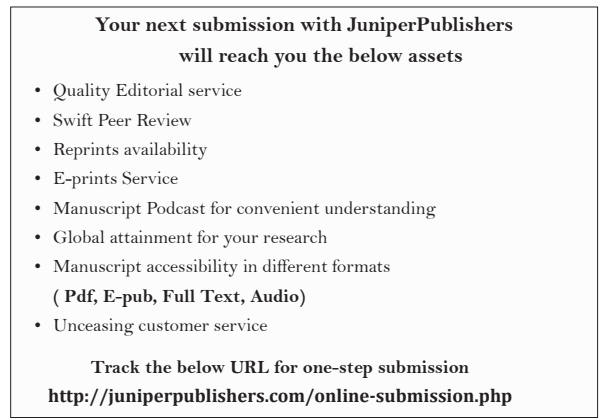

